\title{
Myocardial fibrosis in congenital and pediatric heart disease (Review)
}

\author{
JING TIAN, XINJIANG AN and LING NIU \\ Department of Cardiology, Xuzhou Children's Hospital, Xuzhou, Jiangsu 221002, P.R. China
}

Received June 13, 2016; Accepted February 7, 2017

DOI: 10.3892/etm.2017.4224

\begin{abstract}
Cardiac fibrosis is a common phenomenon in different types of heart diseases, such as ischemic heart disease, inherited cardiomyopathy mutations, diabetes, and ageing and is associated with morbidity and mortality. Increased accumulation of extracellular matrix (ECM) that impacts cardiac function, is the underlying cause of fibrotic heart disease. There are four different types of cardiac fibrosis, including, reactive interstitial fibrosis, replacement fibrosis, infiltrative interstitial fibrosis and endomyocardial fibrosis. They are involved in the activation and transformation of cardiac fibroblasts to myofibroblasts, which participate in ECM production and fibrotic process and several inflammatory pathways. Besides the ECM proteins, myofibroblasts also express smooth muscle $\alpha$-actin, SM22 and caldesmon and other markers related to fibrotic process. Most commonly employed techniques to assess myocardial fibrosis include stress echocardiography, cardiac magnetic resonance imaging and positron emission tomography. Because of the involvement of renin-angiotensin-II-aldosterone system, transforming growth factor- $\beta$ signaling and activin-linked kinase 5 in the mechanisms of cardiac fibrosis, these pathways and the involved proteins are useful as therapeutic targets. However, because of the importance of these pathways in many other physiological functions, their therapeutic targeting needs to be approached with caution.
\end{abstract}

\section{Contents}

1. Introduction

2. Types of cardiac fibrosis

3. Fibroblasts, myofibroblasts and fibrosis

4. Transformation of fibroblasts to myofibroblasts

Correspondence to: Dr Xinjiang An, Department of Cardiology, Xuzhou Children's Hospital, 18 Sudibei Road, Xuzhou, Jiangsu 221002, P.R. China

E-mail: anxinjian001@163.com

Key words: cardiac fibrosis, inherited cardiomyopathy, extracellular matrix
5. Assessment of myocardial fibrosis

6. Therapeutic approaches to treat cardiac fibrosis

7. Conclusion

\section{Introduction}

Cardiac fibrosis is a common histopathological occurrence with many types of heart diseases, including ischemic heart disease, inherited cardiomyopathy mutations, diabetes, and ageing and is associated with morbidity and mortality. While there are several mechanisms of myocardial fibrosis with acute and chronic etiologies, increased accumulation of extracellular matrix (ECM) that impacts cardiac function, is the underlying cause of fibrotic heart disease. Besides the disturbances in the mechanical functions of heart, fibrosis mediated scarring and electrical dysfunction often initiates ventricular arrhythmias, which in turn accelerates events towards heart failure and then sudden cardiac death (1-3). Myocardial fibrosis presents one of the major challenges clinically not just to improve the survival rate and also the quality of life. Cardiac magnetic resonance (CMR) has been useful in measuring the extent of diffuse myocardial fibrosis, which is considered as a common pathological pathway that leads to the loss of myocardial function (4,5). Inasmuch as fibrosis can be reversible (6), its quantification using CMR can be useful in changing the way the cardiac fibrosis patients are monitored and treated (7). Myocardial fibrosis has been demonstrated in the pressure-loaded left ventricle of infants and children with aortic stenosis and coarctation (8). Histological studies revealed right ventricular myofiber disorganization and interstitial fibrosis in patients with tetralogy of Fallot (9). Right ventricular fibrosis is not just found in late adult survivors, but actually is already present in infants suffering with this condition (10). Alterations in myocardial architecture associated with fibrotic remodeling lead to deranged heart function in different ways. Thus, in patients after tetralogy of Fallot repair, fibrous endocardial thickening of the right ventricular infundibulum is a predictor of poor right ventricular function (5). On the other hand, myocardial fibrosis is associated with systolic ventricular dysfunction early in life in patients with tricuspid atresia (11).

Healthy myocardium consists of mostly cardiomyocytes $(\sim 75 \%)$, with the remaining partition, the interstitium regions comprising of fibroblasts, endothelial cells, and coronary arteries (12). The fibroblasts participate in the continuous 
formation and degradation of ECM, which is composed of types I and III collagen fibers, that act as the scaffold for the myocardium. Disturbances in the balance between collagen production and degradation in the ECM, that led to expansion of collagens, cause myocardial fibrosis. Such derangement occurs either due to the death of cardiomyocytes, or because of stimuli that trigger elevated collagen synthesis (13).

\section{Types of cardiac fibrosis}

There are multiple types of cardiac fibrosis depending upon location and causes. i) Reactive interstitial fibrosis (RIF): This is characterized by an increase in collagen synthesis without much effect on the viability of myocardium (14). RIF is considered as a type of myocardial remodeling and leads to an increased interstitial compartment volume without any associated changes in myocyte volume. This type of fibrosis is associated with diffused deposition of collagen and occurs in response to increased pressure and/or volume loads as in the cases of hypertension, aortic stenosis, chronic regurgitation, and shunt, ischemia, hyperglycemia, or ageing. RIF, which generally presents with a progressive chronic course, is possibly reversible by curtailing the damage causing stimuli or even by targeted therapy. ii) Replacement fibrosis: An increased type I collagen deposition and expansion of ECM, following cardiomyocyte death contributes to this type of fibrosis (14). The elevated levels of collagen fibers replace the dead cardiomyocytes, but lack the contractile and electric functionality. Acute or chronic conditions that trigger cardiomyocyte death such as myocardial ischemia and events that damage the cardiomyocyte membrane integrity causing cell death, lead to diffused or focal replacement fibrosis. Unlike the RIF, in replacement fibrosis, the affected myocardium is not viable and thus is unable to recover contractile properties following revascularization or by blocking the damaging process. iii) Infiltrative interstitial fibrosis: This fibrosis is seen in conditions such as amyloidosis or Anderson-Fabry disease (14). Inflammatory cell infiltration was proposed to be an important factor underlying the interstitial fibrosis seen in right ventricles of systemic sclerosis-associated pulmonary arterial hypertension (15). iv) Endomyocardial fibrosis (EMF): EMF affects children under age of 2 years in tropical and subtropical regions, and involves the apical endocardium of either or both right and left ventricles. It has been suggested that EMF as one of the primary causes of pediatric congestive heart failure, which is often overlooked clinically (16). There is no clear etiology for EMF, but hypereosinophilia, infections, autoimmunity, genetic factors and nutritional deficiencies may play a role. EMF has been described to present in three phases: Initial phase of acute carditis with febrile illness and with heart failure in severe cases; an intermediary subacute phase followed by a chronic burnt-out phase (17). Notably, EMF is identified mostly at late stages of the disease and is not observed in the early phase of the disease. However, following diagnosis, there is rapid onset of associated complications such as atrial fibrillation, thromboembolism and progressive atrioventricular valve regurgitation. EMF patients display clinical features of heart failure, with enlargement of both atria with normally functioning ventricles during echocardiography (18). Currently, the approach to treat EMF patients is by surgery, involving targeted endocardial resection combined with valve repair or replacement, as there is no specific medical treatment (16).

\section{Fibroblasts, myofibroblasts and fibrosis}

Although the biology of cardiomyocyte and its apoptotic or necrotic death have been the focus of several investigations addressing cardiac diseases, increasing evidence suggests cardiac fibroblasts in the pathogenic mechanisms $(19,20)$. Fibroblasts, which are abundant in the normal myocardium, have a structural function by providing support (21). However, when these normally quiescent fibroblasts are activated, they transdifferentiate into myofibroblasts, which appear similar to a hybrid of fibroblast and smooth muscle cells. Myofibroblasts are able to effectively secrete and myofibroblasts are absent in healthy heart, they appear within days following a cardiac injury (22). Myofibroblasts appear as spindle-shaped and possess dendritic-like processes (23) that protrude from the cell body. Characteristic elongated and serrated nuclei, extensive areas of rough endoplasmic reticulum and irregular non-sarcomeric myofibrillar structures are morphological features of myofibroblasts as observed in electron micrographs of pressure-overloaded rat heart cross sections (24). Specific protein markers of myofibroblasts are mainly the ECM proteins, such as periostin, collagens I and III and fibronectin (25). Besides these ECM proteins, myofibroblasts also express smooth muscle $\alpha$-actin, SM22 and caldesmon $(26,27)$. Of note, most of the myofibroblasts present in the border zone and approximately half of those in the fibrotic lesions of damaged myocardium, express embryonic smooth muscle myosin and $\alpha$-SMA but not other smooth muscle markers, which is a major difference from smooth muscle cells (28).

\section{Transformation of fibroblasts to myofibroblasts}

It has been suggested that there are two stages in the transformation of myofibroblast: in the first stage, there is development of 'proto-myofibroblasts' from fibroblasts, with the characteristic formation of cytoplasmic actin stress fibers and small adhesion complexes, which facilitate the migration of these cells to the injured myocardium, where these cells secrete collagens and fibronectin and assume physical orientation in order to align themselves with the primary stress axes of the injured tissue (25). In the second stage, which starts approximately 20-30 h following cardiac injury, the cytokines and mechanical stress convert these proto-myofibroblasts to mature myofibroblasts, which express $\alpha$-SMA, which forms the stress fiber network (25). With further maturation of the myofibroblast, besides the $\alpha$-SMA, cadherin- 11 is also secreted and adds to the strength of the stress fiber network (29) (Fig. 1). Normally myofibroblasts have a protective function in the heart by participating acutely in injury repair response. Although uninterrupted fibrosis can be dangerous to heart, the function of myofibroblasts is important to maintain functional myocardium following any stress insult. Thus, following myocardial infarction, when cardiomyocytes die over several days due to ischemia, myofibroblasts play a major role in the remodeling of a fibrotic scar in the affected myocardium, in order to prevent wall rupture (30,31). Myofibroblasts eventually disappear, through less understood mechanisms, in many 
Cardiac injury/ insult and Myocardial fibrosis

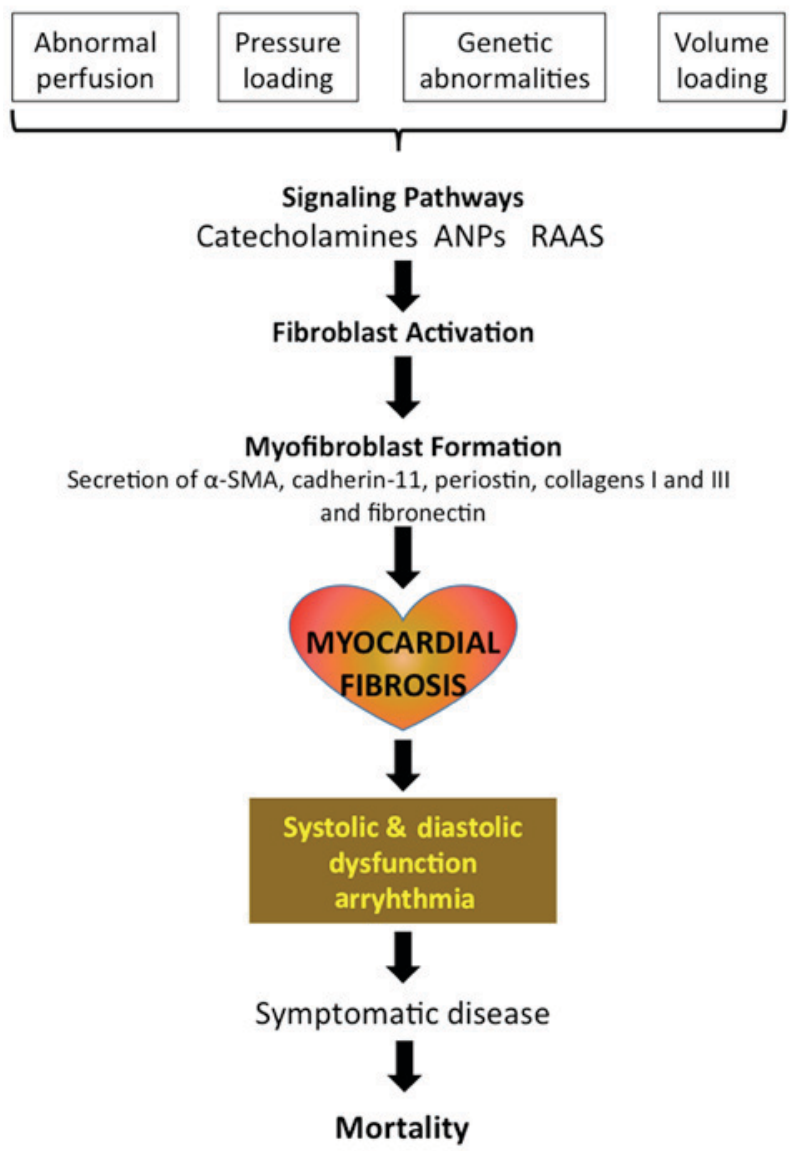

Figure 1. Insult and injury to heart lead to cardiac fibrosis. Several insults such as pressure and volume loading and even certain genetic abnormalities lead to the activation of different signaling pathways such as RAAS, catecholamines and natriuretic peptides. These pathways cause activation/transformation of cardiac fibroblasts to myofibroblasts, which participate in elevated ECM deposition and myocardial fibrosis. Fibrosis disturbance causes diastolic and systolic function and causes arrhythmia, all of which are clinical features of heart failure. RAAS, renin-angiotensin-aldosterone system; ECM, extracellular matrix.

situations of wound healing. One possible mechanism is that following the formation of permanent scar and stabilization of cardiac injury, which may take up to 2 weeks, a significant proportion of the myofibroblasts undergo senescence (32) and/or apoptosis (33). It has also been proposed that some of the myofibroblasts may dedifferentiate back to fibroblast phenotype, even though strong evidence for this is lacking in myocardium. Critical for the pathogenesis of cardiac fibrosis is the persistent presence of myofibroblasts in the myocardium, long after the resolution of stress insult (Fig. 1). This is generally either due to concurrent dysregulation of neuroendocrine factors or even mechanical stress, both of which lead to and aggravate chronic cardiac fibrosis and/or hypertrophic scarring $(34,35)$. A common example for this is the intermittent ischemia, chronically affecting the heart, creating constant wound healing milieu, which leads to persistent activation and formation of the myofibroblasts and the eventual fibrosis (30). It has been proposed that the function of resident fibroblasts is to maintain normal tissue integrity and structure, whereas, acute injury or altered neuroendocrine signaling stimulates fibroblasts from other sources, which

\section{Signaling pathways involved in cardiac fibrosis}

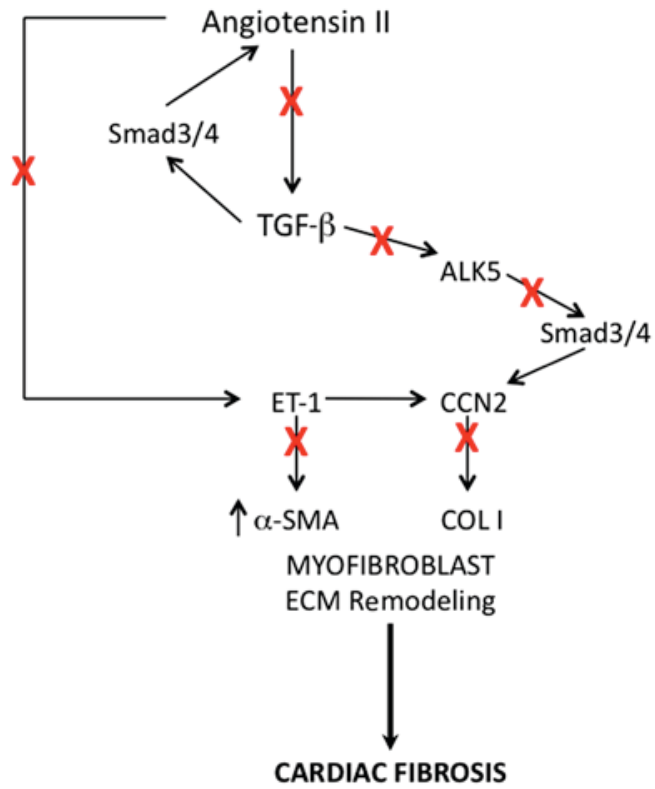

Figure 2. Signaling pathways involved in cardiac fibrosis and possible therapeutic targets. Angiotensin-II induces TGF- $\beta, \mathrm{CCN} 2$, and ET-1 directly; TGF- $\beta$, in turn can induce ET-1 and CCN2; ET-1 can also induce CCN2. There is positive feedback between TGF- $\beta$ and angiotensin-II through the induction of angiotensin receptor. TGF- $\beta$ signaling involves Smads 3 or -4 . All these signaling components promote fibroblast activation to myofibroblast, which plays a key role in fibrosis through the secretion of extracellular matrix proteins and $\alpha$-SMA. These signaling components make potential therapeutic targets (indicated with red X) for preventing cardiac fibrosis. TGF- $\beta$, transforming growth factor- $\beta$; ET-1, endothelin-1.

ultimately contribute to pathophysiologic fibrosis $(36,37)$. A better understanding of the molecular mechanisms involved in cardiac fibrosis and the regulation of the players involved such as myofibroblast formation and activation, is essential for developing effective therapeutics.

\section{Assessment of myocardial fibrosis}

The most commonly employed techniques in patients with congenital heart disease to assess the fibrotic features of myocardium include stress echocardiography and CMR and also positron emission tomography (PET), which is the reference standard for monitoring myocardial viability. Stress echocardiography allows for the assessment of myocardial contractile reserve in patients with adequate acoustic windows. Because of this, the main limitation of stress echocardiography is decreased diagnostic accuracy in patients with poor acoustic windows (38). PET scan is a nuclear scintigraphy and employs ${ }^{13} \mathrm{~N}$-labeled ammonia, which a marker for coronary perfusion and ${ }^{18} \mathrm{~F}$-fluorodeoxyglucose, a glucose surrogate, for monitoring myocardial metabolism. A mismatch between the imaging of coronary perfusion and metabolism is indicative of nonviable tissue with the possibility of replacement fibrosis. However, PET scanner availability is limited and its usage requires an on-site cyclotron, and PET is not sensitive enough to detect interstitial fibrosis (39). CMR is the major technique used to collect data on cardiac fibrosis. CMR is able to provide much better spatial resolution and visualization of all myocardial segments, in the absence of any contaminating/interfering metallic particles, and 
this technique is used extensively for the quantitative assessment of ventricular size and systolic function. There are two major CMR approaches for assessing myocardial fibrosis: Late gadolinium enhancement (LGE) imaging and T1 mapping/ECV fraction calculation. LGE identifies areas of discrete replacement fibrosis, with high sensitivity and specificity (13).

\section{Therapeutic approaches to treat cardiac fibrosis}

In the normal healthy heart, collagen network, which contributes to ECM and maintains myocardial architecture as well as chamber geometry, also provides tensile strength and elasticity, the necessary factors for normal systolic and diastolic function of ventricles (40). Several local and hormonal systems, such as renin-angiotensin-aldosterone system (RAAS), regulate the dynamic collagen turnover, and disturbances in these systems lead to an imbalance between collagen synthesis and degradation with the resultant accumulation of collagen, the underlying factor of cardiac fibrosis, followed by deranged myocardial architecture (41). Thus it has been shown that pharmacological intervention of RAAS could prevent this process of fibrosis and at times even reverse the damage caused (42). Thus, aldosterone antagonism with spironolactone has been found to lower collagen levels in hearts, ex vivo (43), improve diastolic function (44), decrease left ventricular size and ejection fraction (45), and also mortality (Fig. 2) (46).

There is ample evidence indicating increased expression of transforming growth factor- $\beta$ (TGF- $\beta$ ) in response to injury and TGF- $\beta$ is known to play an important role during fibroblast activation and myofibroblast differentiation, and also fibrosis (Fig. 2) (47,48). TGF- $\beta$ acts via binding to its cell surface receptor, activin linked kinase 5 (ALK5), which phosphorylates Smad2 and -3. Phospho-Smad2 and -3 bind to Smad4, which translocates into the nucleus, leading to the activation of target gene transcription. It has been shown that inhibitors of ALK5 signaling block certain steps of fibrosis process and thus there is a potential that ALK5 can be a therapeutic target for cardiac fibrosis (49). Even though neutralizing antibodies against TGF- $\beta$ have been tried in some studies (50), because of involvement of TGF- $\beta$ in several different cellular functions, such approach proved to be not appropriate (51) and ALK5 antagonists are much better choice (52). Angiotensin II has been shown to function in concert with TGF- $\beta$ in the signaling pathways that lead to cardiac fibrosis $(53,54)$. Blockade of angiotensin receptor with inhibitors like losartan is effective in decreasing cardiac fibrosis in animal models and also in humans and this approach of angiotensin-II inhibitors seems to be better than blocking TGF- $\beta$ (55).

Similarly, antagonism of endothelin-1, which induces ECM production and myofibroblast transformation (56) was found to reduce cardiac fibrosis. Other possible therapeutic targets include platelet derived growth factor and connective tissue growth factor, CCN2, but further work is needed to ascertain this possibility (57).

\section{Conclusion}

Cardiac fibrosis, which is seen in many types of heart diseases, involves increased accumulation of ECM, mediated by the activated myofibroblasts, arising from cardiac fibroblasts.
Myocardial fibrosis include stress echocardiography and CMR and also PET. Renin-angiotensin-II-aldosterone system, TGF- $\beta$ signaling and ALK5 have been found useful as therapeutic targets. However, because of the importance of these pathways in many other physiological functions, their therapeutic targeting needs to be approached with caution.

\section{References}

1. Harris KM, Spirito P, Maron MS, Zenovich AG, Formisano F, Lesser JR, Mackey-Bojack S, Manning WJ, Udelson JE and Maron BJ: Prevalence, clinical profile, and significance of left ventricular remodeling in the end-stage phase of hypertrophic cardiomyopathy. Circulation 114: 216-225, 2006.

2. O'Hanlon R, Grasso A, Roughton M, Moon JC, Clark S, Wage R, Webb J, Kulkarni M, Dawson D, Sulaibeekh L, et al: Prognostic significance of myocardial fibrosis in hypertrophic cardiomyopathy. J Am Coll Cardiol 56: 867-874, 2010.

3. Vasquez $\mathrm{C}$ and Morley GE: The origin and arrhythmogenic potential of fibroblasts in cardiac disease. J Cardiovasc Transl Res 5: 760-767, 2012.

4. Ho SY, Jackson M, Kilpatrick L, Smith A and Gerlis LM: Fibrous matrix of ventricular myocardium in tricuspid atresia compared with normal heart. A quantitative analysis. Circulation 94: 1642-1646, 1996.

5. Farah MC, Castro CR, Moreira VM, Binotto MA, Guerra VC, Riso AA, Marcial MB, Lopes AA, Mathias W Jr and Aiello VD: The impact of preexisting myocardial remodeling on ventricular function early after tetralogy of Fallot repair. J Am Soc Echocardiogr 23: 912-918, 2010.

6. Burns KM, Byrne BJ, Gelb BD, Kühn B, Leinwand LA, Mital S, Pearson GD, Rodefeld M, Rossano JW, Stauffer BL, et al: New mechanistic and therapeutic targets for pediatric heart failure: Report from a National Heart, Lung, and Blood Institute working group. Circulation 130: 79-86, 2014.

7. Moon JC, Messroghli DR, Kellman P, Piechnik SK, Robson MD, Ugander M, Gatehouse PD, Arai AE, Friedrich MG, Neubauer S, et al; Society for Cardiovascular Magnetic Resonance Imaging; Cardiovascular Magnetic Resonance Working Group of the European Society of Cardiology: Myocardial T1 mapping and extracellular volume quantification: A Society for Cardiovascular Magnetic Resonance (SCMR) and CMR Working Group of the European Society of Cardiology consensus statement. J Cardiovasc Magn Reson 15: 92, 2013.

8. Cheitlin MD, Robinowitz M, McAllister H, Hoffman JI, Bharati S and Lev M: The distribution of fibrosis in the left ventricle in congenital aortic stenosis and coarctation of the aorta. Circulation 62: 823-830, 1980.

9. Chowdhury UK, Sathia S, Ray R, Singh R, Pradeep KK and Venugopal P: Histopathology of the right ventricular outflow tract and its relationship to clinical outcomes and arrhythmias in patients with tetralogy of Fallot. J Thorac Cardiovasc Surg 132: 270-277, 2006.

10. Peters TH, Sharma HS, Yilmaz E and Bogers AJ: Quantitative analysis of collagens and fibronectin expression in human right ventricular hypertrophy. Ann N Y Acad Sci 874: 278-285, 1999.

11. Sanchez-Quintana D, Climent V, Ho SY and Anderson RH: Myoarchitecture and connective tissue in hearts with tricuspid atresia. Heart 81: 182-191, 1999.

12. Baum J and Duffy HS: Fibroblasts and myofibroblasts: What are we talking about? J Cardiovasc Pharmacol 57: 376-379, 2011.

13. Rathod RH, Powell AJ and Geva T: Myocardial fibrosis in congenital heart disease. Circ J 80: 1300-1307, 2016.

14. Mewton N, Liu CY, Croisille P, Bluemke D and Lima JA: Assessment of myocardial fibrosis with cardiovascular magnetic resonance. J Am Coll Cardiol 57: 891-903, 2011.

15. Overbeek MJ, Mouchaers KT, Niessen HM, Hadi AM, Kupreishvili K, Boonstra A, Voskuyl AE, Belien JA, Smit EF, Dijkmans BC, et al: Characteristics of interstitial fibrosis and inflammatory cell infiltration in right ventricles of systemic sclerosis-associated pulmonary arterial hypertension. Int J Rheumatol 2010: 604615, 2010.

16. Rohit M, Gupta A and Talwar KK: Heart failure in children in tropical regions. Curr Heart Fail Rep 10: 277-284, 2013.

17. Ball JD, Williams AW and Davies JN: Endomyocardial fibrosis. Lancet 266: 1049-1054, 1954. 
18. Ammash NM, Seward JB, Bailey KR, Edwards WD and Tajik AJ: Clinical profile and outcome of idiopathic restrictive cardiomyopathy. Circulation 101: 2490-2496, 2000.

19. Krenning G, Zeisberg EM and Kalluri R: The origin of fibroblasts and mechanism of cardiac fibrosis. J Cell Physiol 225: 631-637, 2010.

20. Turner NA and Porter KE: Function and fate of myofibroblasts after myocardial infarction. Fibrogenesis Tissue Repair 6: 5, 2013.

21. Banerjee I, Yekkala K, Borg TK and Baudino TA: Dynamic interactions between myocytes, fibroblasts, and extracellular matrix. Ann N Y Acad Sci 1080: 76-84, 2006.

22. Sun Y and Weber KT: Infarct scar: A dynamic tissue. Cardiovasc Res 46: 250-256, 2000.

23. Eyden B: The myofibroblast: Phenotypic characterization as a prerequisite to understanding its functions in translational medicine. J Cell Mol Med 12: 22-37, 2008.

24. Shiojima I, Aikawa M, Suzuki J, Yazaki Y and Nagai R: Embryonic smooth muscle myosin heavy chain SMemb is expressed in pressure-overloaded cardiac fibroblasts. Jpn Heart J 40: 803-818, 1999.

25. Tomasek JJ, Gabbiani G, Hinz B, Chaponnier C and Brown RA: Myofibroblasts and mechano-regulation of connective tissue remodelling. Nat Rev Mol Cell Biol 3: 349-363, 2002.

26. Hinz B: Formation and function of the myofibroblast during tissue repair. J Invest Dermatol 127: 526-537, 2007.

27. Hinz B: The myofibroblast: Paradigm for a mechanically active cell. J Biomech 43: 146-155, 2010.

28. Frangogiannis NG, Michael LH and Entman ML: Myofibroblasts in reperfused myocardial infarcts express the embryonic form of smooth muscle myosin heavy chain (SMemb). Cardiovasc Res 48: 89-100, 2000

29. Hinz B, Pittet P, Smith-Clerc J, Chaponnier C and Meister JJ: Myofibroblast development is characterized by specific cell-cel adherens junctions. Mol Biol Cell 15: 4310-4320, 2004

30. Weber KT, Sun Y, Bhattacharya SK, Ahokas RA and Gerling IC: Myofibroblast-mediated mechanisms of pathological remodelling of the heart. Nat Rev Cardiol 10: 15-26, 2013.

31. van den Borne SW, Isobe S, Verjans JW, Petrov A, Lovhaug D, Li P, Zandbergen HR, Ni Y, Frederik P, Zhou J, et al: Molecular imaging of interstitial alterations in remodeling myocardium after myocardial infarction. J Am Coll Cardiol 52: 2017-2028, 2008.

32. Krizhanovsky V, Yon M, Dickins RA, Hearn S, Simon J, Miething C, Yee H, Zender L and Lowe SW: Senescence of activated stellate cells limits liver fibrosis. Cell 134: 657-667, 2008.

33. Kissin E and Korn JH: Apoptosis and myofibroblasts in the pathogenesis of systemic sclerosis. Curr Rheumatol Rep 4: 129-135, 2002

34. Bataller R and Brenner DA: Liver fibrosis. J Clin Invest 115: 209-218, 2005

35. Scotton CJ and Chambers RC: Molecular targets in pulmonary fibrosis: The myofibroblast in focus. Chest 132: 1311-1321, 2007.

36. Crawford JR, Haudek SB, Cieslik KA, Trial J and Entman ML: Origin of developmental precursors dictates the pathophysiologic role of cardiac fibroblasts. J Cardiovasc Transl Res 5: 749-759, 2012.

37. Biernacka A and Frangogiannis NG: Aging and cardiac fibrosis. Aging Dis 2: 158-173, 2011.

38. Camici PG, Prasad SK and Rimoldi OE: Stunning, hibernation, and assessment of myocardial viability. Circulation 117: 103-114, 2008.

39. Auerbach MA, Schöder H, Hoh C, Gambhir SS, Yaghoubi S, Sayre JW, Silverman D, Phelps ME, Schelbert HR and Czernin J: Prevalence of myocardial viability as detected by positron emission tomography in patients with ischemic cardiomyopathy. Circulation 99: 2921-2926, 1999.
40. Delcayre C and Swynghedauw B: Molecular mechanisms of myocardial remodeling. The role of aldosterone. J Mol Cell Cardiol 34: 1577-1584, 2002

41. Weber KT and Brilla CG: Pathological hypertrophy and cardiac interstitium. Fibrosis and renin-angiotensin-aldosterone system. Circulation 83: 1849-1865, 1991.

42. Cohn JN: Myocardial structural effects of aldosterone receptor antagonism in heart failure. J Am Coll Cardiol 50: 597-599, 2007.

43. Suzuki G, Morita H, Mishima T, Sharov VG, Todor A, Tanhehco EJ, Rudolph AE, McMahon EG, Goldstein S and Sabbah HN: Effects of long-term monotherapy with eplerenone, a novel aldosterone blocker, on progression of left ventricular dysfunction and remodeling in dogs with heart failure. Circulation 106: 2967-2972, 2002.

44. Izawa H, Murohara T, Nagata K, Isobe S, Asano H, Amano T, Ichihara S, Kato T, Ohshima S, Murase Y, et al: Mineralocorticoid receptor antagonism ameliorates left ventricular diastolic dysfunction and myocardial fibrosis in mildly symptomatic patients with idiopathic dilated cardiomyopathy: A pilot study. Circulation 112: 2940-2945, 2005.

45. Cicoira M, Zanolla L, Rossi A, Golia G, Franceschini L, Brighetti G, Marino P and Zardini P: Long-term, dose-dependent effects of spironolactone on left ventricular function and exercise tolerance in patients with chronic heart failure. J Am Coll Cardiol 40: 304-310, 2002.

46. Pitt B, Zannad F, Remme WJ, Cody R, Castaigne A, Perez A, Palensky J and Wittes J; Randomized Aldactone Evaluation Study Investigators: The effect of spironolactone on morbidity and mortality in patients with severe heart failure. N Engl J Med 341: 709-717, 1999.

47. Massagué J: TGF-beta signal transduction. Annu Rev Biochem 67: 753-791, 1998.

48. Leask A and Abraham DJ: TGF-beta signaling and the fibrotic response. FASEB J 18: 816-827, 2004.

49. Chen Y, Xu SW, Eastwood M, Black CM, Denton CP, Leask A and Abraham DJ: Contribution of activin receptor-like kinase 5 (transforming growth factor beta receptor type I) signaling to the fibrotic phenotype of scleroderma fibroblasts. Arthritis Rheum 54: 1309-1316, 2006.

50. Kuwahara F, Kai H, Tokuda K, Kai M, Takeshita A, Egashira K and Imaizumi T: Transforming growth factor-beta function blocking prevents myocardial fibrosis and diastolic dysfunction in pressure-overloaded rats. Circulation 106: 130-135, 2002.

51. Frantz S, Hu K, Adamek A, Wolf J, Sallam A, Maier SK, Lonning S, Ling H, Ertl G and Bauersachs J: Transforming growth factor beta inhibition increases mortality and left ventricular dilatation after myocardial infarction. Basic Res Cardiol 103: 485-492, 2008.

52. Tan SM, Zhang Y, Connelly KA, Gilbert RE and Kelly DJ: Targeted inhibition of activin receptor-like kinase 5 signaling attenuates cardiac dysfunction following myocardial infarction. Am J Physiol Heart Circ Physiol 298: H1415-H1425, 2010.

53. Campbell SE and Katwa LC: Angiotensin II stimulated expression of transforming growth factor-beta1 in cardiac fibroblasts and myofibroblasts. J Mol Cell Cardiol 29: 1947-1958, 1997.

54. Schultz JJ, Witt SA, Glascock BJ, Nieman ML, Reiser PJ, Nix SL, Kimball TR and Doetschman T: TGF-betal mediates the hypertrophic cardiomyocyte growth induced by angiotensin II. J Clin Invest 109: 787-796, 2002

55. De Mello WC and Specht P: Chronic blockade of angiotensin II AT1-receptors increased cell-to-cell communication, reduced fibrosis and improved impulse propagation in the failing heart. J Renin Angiotensin Aldosterone Syst 7: 201-205, 2006.

56. Leask A: Targeting the TGFbeta, endothelin- 1 and $\mathrm{CCN} 2$ axis to combat fibrosis in scleroderma. Cell Signal 20: 1409-1414, 2008.

57. Leask A: Potential therapeutic targets for cardiac fibrosis: TGFbeta, angiotensin, endothelin, CCN2, and PDGF, partners in fibroblast activation. Circ Res. 106: 1675-1680, 2010. 